Gut and Liver, Vol. 10, No. 6, November 2016, pp. 869-870

\title{
Comparative Effectiveness of Lamivudine versus Entecavir in Patients with Hepatocellular Carcinoma: Watch out for Confounders!
}

\author{
Hwi Young Kim \\ Department of Internal Medicine, Ewha Womans University School of Medicine, Seoul, Korea
}

See "Lamivudine versus Entecavir for Newly Diagnosed Hepatitis B Virus-Related Hepatocellular Carcinoma" by Jung Hee Kim, et al. on page 939, Vol. 10. No. 6, 2016

Recently, comparative effectiveness research (CER) has received attention as an underestimated yet valuable research methodology in the field of liver diseases. ${ }^{1}$ CER is particularly useful for research questions for which prospective randomized trials are not feasible due to time, cost or ethical issues. Furthermore, cost-effectiveness or economic aspect of health problems may also be included in CER. The downsides of CER, however, sometimes weaken the level of evidence from those studies; for example, unidentified biases and confounders. Although using electronic medical records of a large patient population has facilitated CER, biases and confounders may significantly limit the credibility of such studies, especially retrospective ones. The methodological countermeasures to overcome these shortcomings include setting an appropriate comparison group to investigate the effectiveness of a new intervention and securing adequate statistical power.

Management of patients with chronic hepatitis B (CHB) has shown remarkable advances in the last decade, most importantly by virtue of the development and clinical use of antiviral agents. For example, risk of decompensation and development of hepatocellular carcinoma (HCC) was reduced with prolonged virological response under long-term antiviral therapy. ${ }^{2}$ In this issue of Gut and Liver, Kim et al. ${ }^{3}$ covers an intriguing topic on the choice of antiviral agent for patients with hepatitis $\mathrm{B}$ virus (HBV)-related HCC in their large-scale cohort study. In this retrospective CER, the authors concluded that entecavir (ETV) was superior to lamivudine (LAM) in newly diagnosed HBVrelated HCC patients who did not receive antiviral therapy prior to diagnosis, in terms of overall survival, decompensation-free survival, and recurrence-free survival. Wong et al. ${ }^{4}$ reported that
ETV-treated patients with cirrhosis achieved reduction in the risk of hepatic events, HCC development, liver-related and allcause mortality. A retrospective study from Korea also reported lower risk of death or transplantation with ETV compared with LAM. ${ }^{5}$ Apart from the role of antiviral therapy in prevention of liver-related events in patients with $\mathrm{CHB}$ only (i.e., prior to the development of HCC), the endpoints of Kim et al.'s study ${ }^{3}$ included survival, decompensation and recurrence in patients with HBV-related HCC; Were all these endpoints appropriate to be analyzed? To answer this question, it needs to be reminded that the study subjects had two distinct yet interacting diseases, i.e., chronic liver disease (mostly cirrhosis) plus malignancy (HCC).

A recent study by Wong et al. ${ }^{6}$ investigated the effect of antiviral treatment with nucleos(t)ide analogues (NAs) on the outcome of HCC patients after different treatments. In this large-scale retrospective study $(n=2,198)$, beneficial effect of NA treatment was most prominent in patients who underwent surgical resection by reducing recurrence risk (adjusted hazard ratio [HR], 0.58) while the risk reduction was not significant in subgroups with local ablation, transarterial chemoembolization or combination treatments. Regarding the survival benefit, tendency towards better survival was observed in NAtreated patients compared with NA-untreated patients, without statistical significance. Although this study from Hong Kong did not primarily intend to compare the relative effectiveness between different NAs, results of subgroup analysis comparing LAM versus ETV were also included, showing that reduction in postoperative recurrence was greater with ETV than LAM (HR, $0.64 ; p=0.008$ ); however, no difference was observed in survival benefit between patients treated with either one of these two

Correspondence to: Hwi Young Kim

Department of Internal Medicine, Liver Center, Ewha Womans University Mokdong Hospital, Ewha Womans University School of Medicine, 1071 Anyangcheon-ro, Yangcheon-gu, Seoul 07985, Korea

Tel: +82-2-2650-5868, Fax: +82-2-2650-2837, E-mail: hwiyoung@ewha.ac.kr pISSN 1976-2283 eISSN 2005-1212 https://doi.org/10.5009/gnl16476

@ This is an Open Access article distributed under the terms of the Creative Commons Attribution Non-Commercial License (http://creativecommons.org/licenses/by-nc/4.0) which permits unrestricted non-commercial use, distribution, and reproduction in any medium, provided the original work is properly cited. 
NAs. The reason why the risk reduction was observed only in patients who underwent surgical resection for their presumably early-stage HCC might be the explained by tumor factors of the patients with more advanced-stage HCC which would offset any differences in the beneficial effect of two NAs. In the similar context, Kim et al.'s study ${ }^{3}$ found that survival benefit of ETV was not observed in patients with advanced HCC, possibly because of the relative significance of tumor factor rather than the benefit of NAs on the liver function.

More importantly, we should be reminded that the composite endpoints such as disease-free survival and recurrence-free survival are vulnerable endpoints in the research of HCC, considering that death from natural history of underlying liver disease (or cirrhosis) might influence adversely as a confounder in the detection of potential benefits from certain treatments. ${ }^{7}$ This Kim et al.'s study ${ }^{3}$ adapted overall survival, decompensationfree survival and recurrence-free survival as their end-outcome variables. In that sense, overall recurrence and survival analysis including causes of death would have been more appropriate outcome measurements in this study instead of the composite endpoints. In addition, other confounders might have influenced the different outcomes between LAM- and ETV-treated patients, such as various laboratory parameters, comorbid status, concurrent medications which, apparently, were not considered in the analysis. There are also other potential confounders which might have contributed to the recurrence of HCC, including lifestyle parameters such as smoking and alcohol ingestion, family history of malignancies, obesity, and so forth. Although these potential confounders were not fully taken into account in the multivariable model, it is appreciable that the authors tried to adjust potential biases from the nonrandom treatment allocation using propensity scores. Adequately powered study design and vigorous efforts to minimize biases or confounders can reinforce the reliability in this retrospective type of CER in HCC, given the notorious heterogeneity of HCC of which clinical outcomes are determined by highly complex interactions involving various tumor-related factors, underlying liver disease-related factors, treatment-related factors and many other comorbid conditions.

\section{CONFLICTS OF INTEREST}

No potential conflict of interest relevant to this article was reported.

\section{REFERENCES}

1. El-Serag HB, Talwalkar J, Kim WR. Efficacy, effectiveness, and comparative effectiveness in liver disease. Hepatology 2010;52: 403-407.

2. Moon JC, Kim SH, Kim IH, et al. Disease progression in chronic hepatitis B patients under long-term antiviral therapy. Gut Liver 2015;9:395-404.

3. Kim JH, Sinn DH, Kim K, et al. Lamivudine versus entecavir for newly diagnosed hepatitis B virus-related hepatocellular carcinoma. Gut Liver 2016;10:939-947.

4. Wong GL, Chan HL, Mak CW, et al. Entecavir treatment reduces hepatic events and deaths in chronic hepatitis B patients with liver cirrhosis. Hepatology 2013;58:1537-1547.

5. Lim YS, Han S, Heo NY, Shim JH, Lee HC, Suh DJ. Mortality, liver transplantation, and hepatocellular carcinoma among patients with chronic hepatitis B treated with entecavir vs lamivudine. Gastroenterology 2014;147:152-161.

6. Wong GL, Tse YK, Chan HL, Yip TC, Tsoi KK, Wong VW. Oral nucleos(t)ide analogues reduce recurrence and death in chronic hepatitis B-related hepatocellular carcinoma. Aliment Pharmacol Ther 2016;43:802-813.

7. Llovet JM, Di Bisceglie AM, Bruix J, et al. Design and endpoints of clinical trials in hepatocellular carcinoma. J Natl Cancer Inst 2008;100:698-711. 